\title{
Exploring the Concept of Cyberpark: What the Experts Think
}

\author{
Paschalis Arvanitidis ${ }^{1(\bowtie)}$ (D), Konstantinos Lalenis ${ }^{1}$ (D), \\ Georgios Artopoulos ${ }^{2}$ (D), and Montserrat Pallares-Barbera ${ }^{3}$ (ID \\ 1 University of Thessaly, Volos, Greece \\ parvanit@uth.gr,klalenis@prd.uth.gr \\ 2 The Cyprus Institute, Nicosia, Cyprus \\ g.artopoulos@cyi.ac.cy \\ ${ }^{3}$ Universitat Autònoma de Barcelona, Cerdanyola, Spain \\ montserrat.pallares@uab.cat
}

\begin{abstract}
The chapter aims to provide a contextualisation of the cyberpark concept as this is perceived by a wide range of experts in public space and information technologies. To do so it makes use of a questionnaire survey conducted with the participants of the CyberParks COST Action, which collected their views on a number of aspects concerning both the mediated and the not mediated public open spaces, such as: their elements and qualities, the facilities they should offer, the activities they should facilitate, the type of space and location that are most suitable to accommodate them, their appropriate size and manner for their development, their target user group and other aspects of their configuration. The analysis brings out the commonalities and differences in experts' views regarding the mediated and the not mediated public open spaces, and, as such, it contributes to specify further the cyberpark concept, mapping out its characteristics and dimensions. This should enrich the ongoing dialogue within the literature and facilitate interactions between relevant, yet fragmented, scientific disciplines in the area to inform the production and appropriation of both mediated and the not mediated public open spaces.
\end{abstract}

Keywords: Experts $\cdot$ Views $\cdot$ Diversity $\cdot$ Survey

\section{Introduction}

The idea of a technologically augmented public open space (POS) has been seen in a very positive way by some people, but with scepticism by others. People perceive parks, plazas, squares, etc. as places to enjoy for leisure, sometimes for work, but rarely as places specifically designed to bring together the 'real' with the 'virtual' and to offer digital interaction and cyber experiences to people. This chapter gets involved in this discussion and attempts to elucidate the concept and characteristics of such a space, termed as 'cyberpark' (Smaniotto Costa et al. 2017). To do so, the chapter makes use of a survey conducted with various experts on the issue. These are the participants of the COST Action TU 1306 CyberParks. The questionnaire that is used has collected 
their views on a number of cyberpark and public space aspects, aiming to bring out the commonalities and differences in their opinion and, as such, to explore the idea and specify the peculiarities and characteristics of cyberpark, in comparison to POS which are not enhanced with information and communications technology (ICT). Analysis of these data is very important given the variety in scientific backgrounds and training of these people (including social scientists, artists, urban designers, information technology professionals, communication specialists, etc.) and consequently the diversity of their views regarding what a cyberpark might actually be.

In a nutshell, the chapter aims to provide, in a more systematic, organized and structured way, a contextualization of the various dimensions of the ICT mediated POS, vis-à-vis POS that are not enhanced with technology, which emerge due to different perspectives, disciplines, backgrounds, etc. of the related experts. Of course, this chapter is not a conclusive piece of work on the issue; it simply presents a first attempt to explore the terrain and to introduce new elements from the expert's perspectives in understanding the concept of cyberpark and the important role ICTs play to POS in the twenty first century.

\section{From CyberParks (COST Action) to Cyberpark (Concept)}

COST Action CyberParks participants have different cultural/national backgrounds and professional experience (in research, policy making, urban design, arts, etc.), and come from various scientific fields (ICTs specialists, spatial planners, urban developers, geographers, sociologists, anthropologists, economists, communication professionals, etc.), having different education and viewpoints on the issues of production, use and management of POS. This is certainly an advantage for a COST Action, since it provides a stimulating environment for sparking ideas, synergies and cross-fertilisation between different scientific disciplines, but it brings out the diversity that exists in the terminology, perspectives and, perhaps, understanding of what cyberpark is or it should be like.

At the beginning of the CyberParks project the concept of cyberpark was relatively vague; a subtle idea that new technologies are able to play an important part in the development of POS into intelligent spaces, where urban (mainly natural) landscape and digital environment blend together to provide services that enhance the quality of life. It is interesting to observe the evolution of the constituent concepts, the interpretations given, and the focus placed by the participants as the project went through. At the kickoff meeting of the project (Brussels on April 2014) the main objective was to:

...create a research platform on the relationship between information and communication technologies (ICTS) and the production and use of public open spaces, and their relevance to sustainable urban development. The impacts of this relationship will be explored from social, ecological, urban design and methodological perspectives. 
This lead, in a second CyberParks meeting (in Barcelona on November 2014) to the provision of the following working definition concerning cyberpark:

A cyberpark is a designed ecosystem of living processes and technologies. It comprises an outdoor green or blue space interacting with a digital intelligent environment. It usually contains living beings, plants, trees, and water features, many of which are integrated with computerised sensors, haptic technologies and virtual objects. Its features can vary widely. For example: It can be used for a range of purposes such as exercise, leisure, social interaction, relaxation and many other activities. It may be public or private. It can be found in urban, suburban, rural, coastal and wilderness areas. It can consist of a large area of land not covered with buildings, roads, or sports facilities, or a dedicated small area in such places as streets, squares, buildings (courtyards, roofs), or disused thoroughfares like the New York Highline. It can also be a beach, ocean, lake, river, or wetland.

We see that the concept evolves to perceive cyberpark not simply as a technologically blended place, but as an ecosystem; the definition deepens on the specifics of the constituent elements, such as outdoor green or blue space; it highlights the ICT connection; it sets forth the diversity of virtual object present in a cyberpark; and emphasizes the flexibility of the concept, to be seen as a working definition which could serve different objectives and agents, from academics to public and private institutions and users. In time, the many understandings of the concept started converging to a more concise meaning of what a cyberpark is:

\begin{abstract}
A cyberpark is a new type of urban landscape where nature and cybertechnologies blend together to generate hybrid experiences and enhance quality of life. The attributes of a Cyberpark (referenced from the Smart Cities initiative) could be defined by the use of sensor technologies in a connectable space, accessible to the public through ubiquitous technologies used in sociable and sharable ways where the virtual is made visible or augments the landscape. ICT can be used in this context to give or gather information, to aid co-creation of space, to allow crowd sourcing of information and opinions, and to allow affective sharing or selfmonitoring of activities. Hardware may be embedded in the environment in the form of responsive sound or lighting systems, control systems, kinetic objects or artworks, passive sensor technologies and display systems. We recognize that the use of such affordances will be qualified by such considerations as the time of day, the duration of the visit, the weather and temperature, location, season, individual or group engagement, age, gender, purpose of visit and the topology and size of the space. (Agora 2017)
\end{abstract}

Currently, it is important to emphasize the complexity of the cyberpark concept and its relation to quality of life in which "the virtual is made visible or augments the landscape." In this sense work is needed to identify cyberpark's specific characteristics and peculiarities that distinguish it from the general category of POS. These are attempted in the following section.

\title{
3 Exploring the Understanding of the Cyberpark Concept
}

\subsection{Research Concept and Methodology}

This section discusses how CyberParks Action participants perceive the notion of cyberpark and its dimensions, vis-à-vis other, more conventional, POS, i.e. POS that 
are not enhanced with technology. Data was collected through a questionnaire survey that explored a number of issues, such as: the elements and qualities of both cyberpark and POS, the facilities they should offer, the activities they should facilitate, the type of space and location that are most suitable to accommodate them, their appropriate size and manner for their development, their target user group and other aspects of their configuration.

Established studies in the area, such as the one by the Project for Public Spaces ${ }^{1}$, have observed that in order to be relevant and occupied (i.e., be sustainable and alive), a POS must:

- Encourage the sociality and socialisation of its users, by being hospitable, friendly, interactive, multi-thematic, collective, familiar, etc.

- Offer a variety of uses and related activities, by being pleasant, lively, unique, useful, local and sustainable.

- Provide comfort and an attractive image, by being safe, clean, "green", providing places to sit and rest but also paths for walking, being pleasant, encouraging intellectual pursuits, being attractive and showcasing local historical moments.

- Be accessible and hospitable to all, by providing continuity with the urban fabric, proximity, connectivity, being easily readable and recognisable, convenient, and by not excluding any social group.

These considerations have been taken into account for the construction of the questionnaire that contributed to this chapter. Also, the authors acknowledge that POS, with its complexities, indeterminacy, and abundance of possible interactions, when it is accessible by the wanderer, local or tourist, offers a sensual experience. This became a point of entry for the development of both CyberParks and cyberpark, as it is further discussed in other chapters of this book. The enriched appropriation of citizens to public space and to data overlaid on physical space, and most importantly, the enhanced capacity for both individual and collective action for the management of the POS, are considered some of the added values over conventional public space.

The questionnaire developed consists of three parts containing 24 questions where participants were asked to choose from a list of options and to evaluate specific characteristics of both cyberpark and not mediated POS, on a scale of 0 (denoting strong disagreement, negative opinion, etc.) to 10 (denoting strong agreement, positive opinion, etc.). In particular, the first part of the questionnaire informs the participants on the purpose of the research and ensures the anonymity of participation. The second part gathers socio-demographic information, such as gender, age, nationality, discipline of specialisation, and occupation. Finally, the third part records views regarding the number of cyberpark's and POS's aspects examined. Survey questions were pre-tested in a pilot study enabling fine-tuning of the instrument.

\footnotetext{
${ }^{1}$ The Project for Public Spaces is a non-profit organisation for study, design and education with the goal of creating sustainable active public and collective spaces for the strengthening of community. The organisation was founded in the United States in 1975 and since then has studied more than 3,000 communities in 43 countries; see <http://www.pps.org/about/>.
} 
The survey was conducted in April 2017 during the CyberParks' Working Groups Meeting. Questionnaires were distributed in person by the members of the research team and asked to be completed on the spot. In order to increase response rate and quality, participants were given the choice of their responses to be recorded by the researcher, or, should they wish, to complete the questions by themselves on their own time. Questionnaires were collected, validated, and then coded and analysed to generate a number of statistics illustrating the respondents' answers on the issues raised.

\subsection{Composition of Respondents}

From a targeted population of 48 experts that participated in the Ancona meeting of the project (where our data were collected), 44 validated questionnaires were acquired (91.7\%). The gender composition of the respondents was about $63.6 \%$ male and $36.4 \%$ female. The average age was about 47 years, with the youngest expert being 30 years old and the oldest 69. The respondents are from 22 countries all over Europe and Israel, but higher participation was from Italy (5 respondents), Portugal (5), Spain (5), Poland (3) and Sweden (3) (see Fig. 1). The vast majority of the respondents $(90.0 \%$ of the sample) hold a $\mathrm{PhD}$, whereas the rest have completed postgraduate studies $(9.1 \%)$. Their knowledge base is informed by 13 academic disciplines (Fig. 2), ranging from spatial planning and urban design, to information technology and various social science subjects (sociology, anthropology, economics, etc.). Most of the participants are academics $(67.4 \%)$, some work in the private sector $(16.3 \%)$, others in the public sector $(11.6 \%)$ and a few are freelancers $(4.7 \%)$.

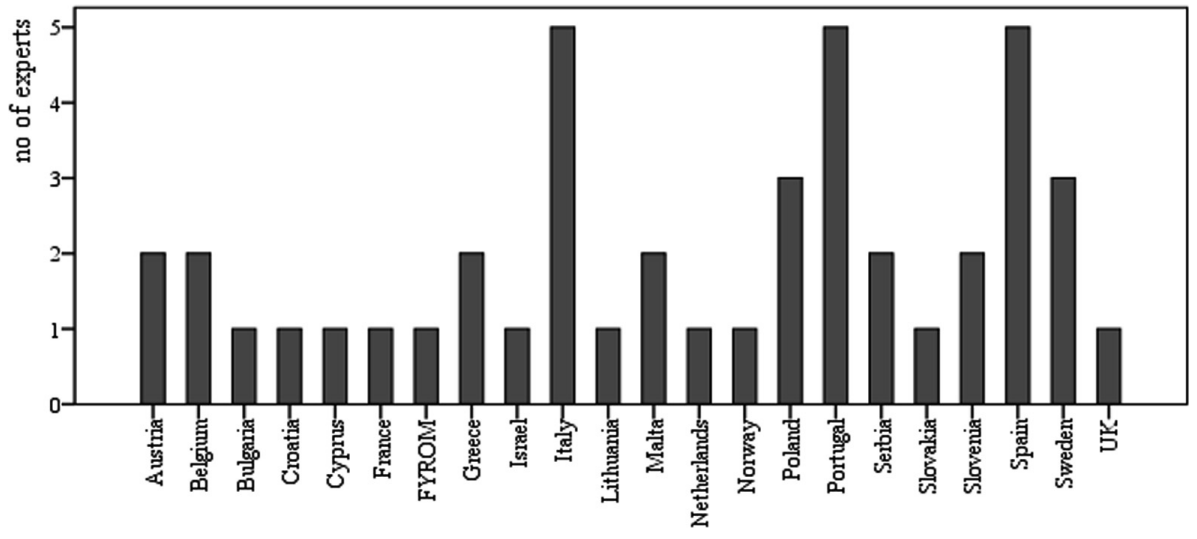

Fig. 1. Origin country of respondents 


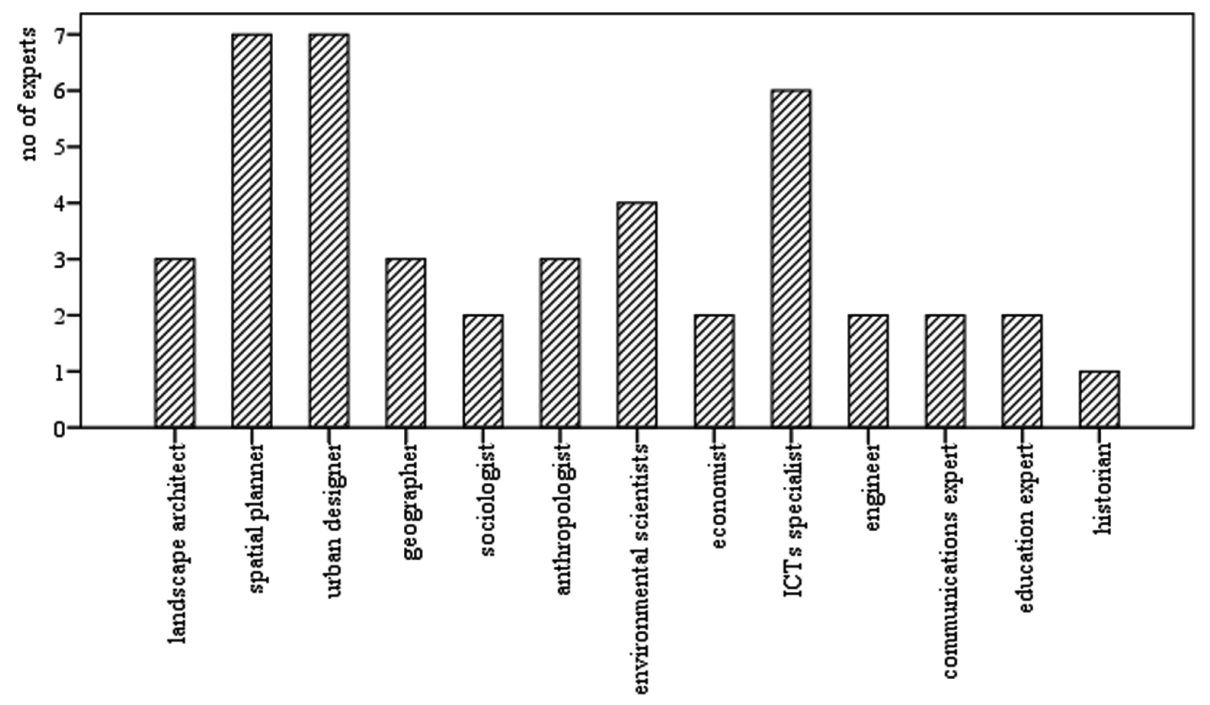

Fig. 2. Specialization of respondents

\subsection{Analysis}

Firstly, the participants were asked to specify which one of the three constituent elements, that is, space, people and technology, is the principal feature of cyberpark and POS. As Fig. 3 shows, experts regard that people is the central element of both, but whereas in POS technology plays a rather minimal role, in cyberpark this becomes the second most important component, followed by space, which comes last in array.

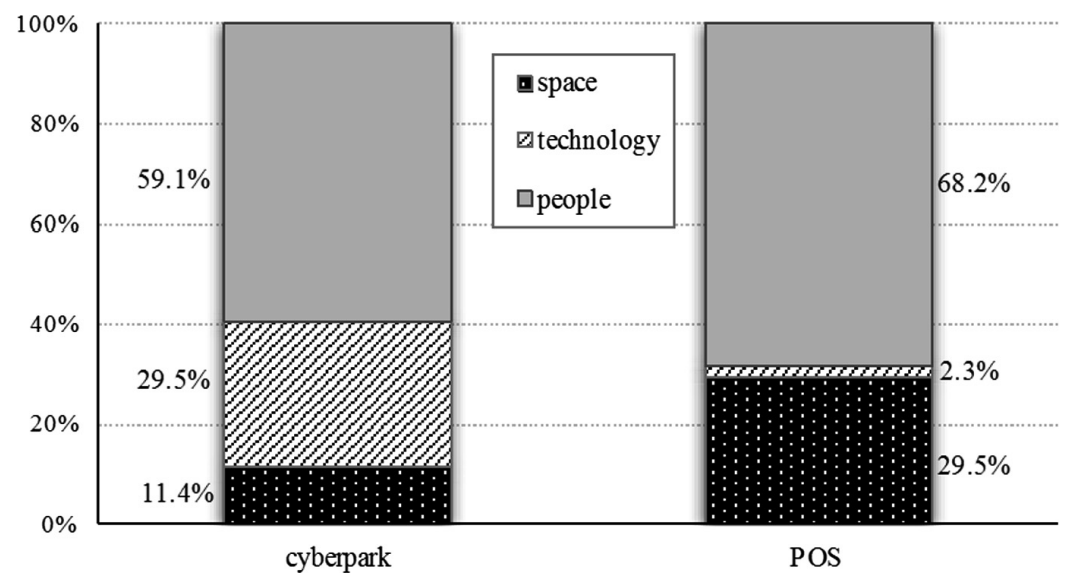

Fig. 3. Importance of constituent elements 
In addition, participants were invited to identify five keywords that best define the concept of cyberpark. Their responses are classified on the basis of the three constituent elements and are presented in Table 1 below. As becomes evident, from the 53 keywords provided by the experts, information and communications technologies (ICTs) constitutes the distinctive one that characterises cyberpark (identified by 23 people). Public space (here mentioned for short as POS) is the second one (identified 14 times), followed by enhanced and interactive space (both identified 9 times). Digital and inclusive space, come next, along with cooperation and community ( 8 times). Figure 4 offers a visualisation of these data, where the importance of each keyword (in terms of the times it has been identified) is represented by its different size in the graph.

Table 1. Cyberpark keywords

\begin{tabular}{l|l|l}
\hline Space & People & Technology \\
\hline POS (14) & Inclusive (8) & ICTs (23) \\
Enhanced (9) & Cooperation (8) & Interactive (9) \\
Innovative (7) & Community (8) & Digital (8) \\
Hybrid (7) & Communication (4) & Wi-fi (5) \\
Open (5) & Well-being (4) & Virtual (4) \\
Green (6) & Connectivity (3) & Apps (3) \\
Quality space (2) & Experience (2) & Information (3) \\
Facilities (2) & Free (2) & Augmented (2) \\
Modern (2) & Users (2) & Smart (2) \\
Added value & Work (2) & Important (2) \\
Diversity & Planning (2) & Inevitable \\
Dynamic & Activities & Sensors \\
Functionality & Bottom-up & Valuable \\
Glocal & Enabling & Energy provision \\
Heterotopia & Engagement & \\
Heuristic & Identity & \\
Temporarity & Learning & \\
Accessibility & Playing & \\
Security & Resilience & \\
& Story-telling & \\
\hline
\end{tabular}

Next, respondents were asked to identify the most important qualities of the spaces under investigation. Figure 5 presents the results. We see that information transmission is perceived to be the most important quality of cyberpark (a feature that is ranked very low in conventional POS), followed by safety and space quality, both of which are regarded as quite important qualities of good POS. Interestingly, the existence of natural environment and greenery is regarded as an essential quality of POS, but not that much of a cyberpark. 


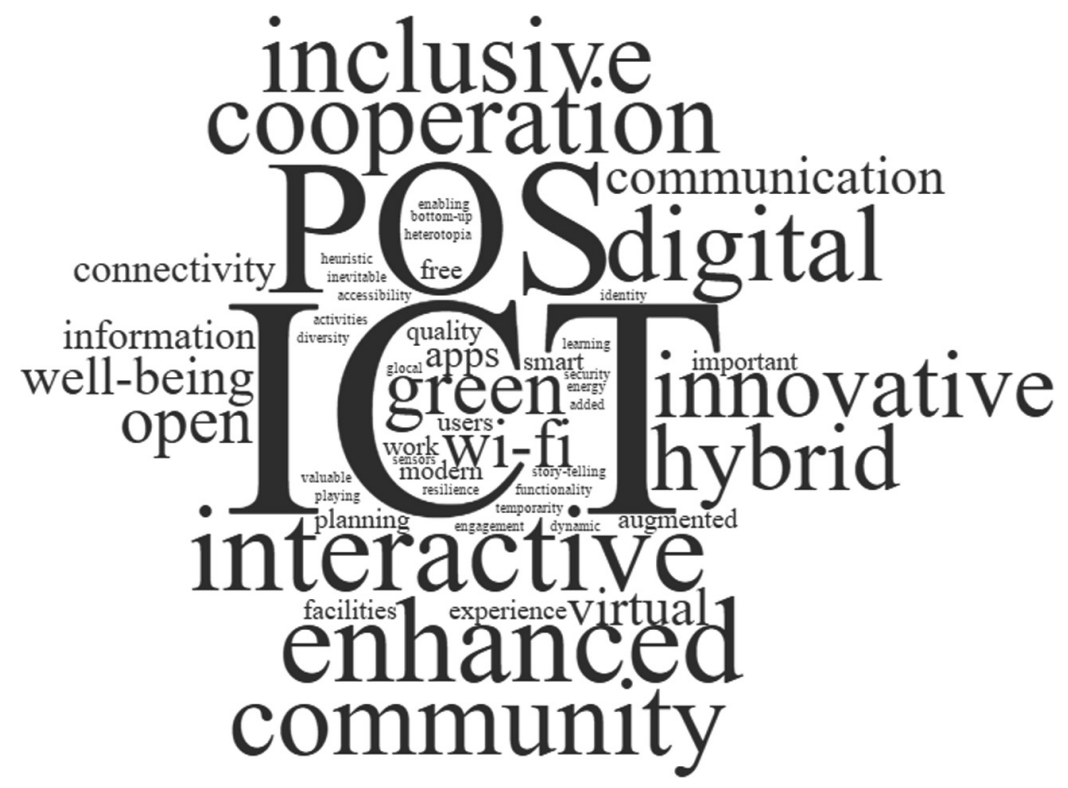

Fig. 4. Word-cloud of cyberpark keywords

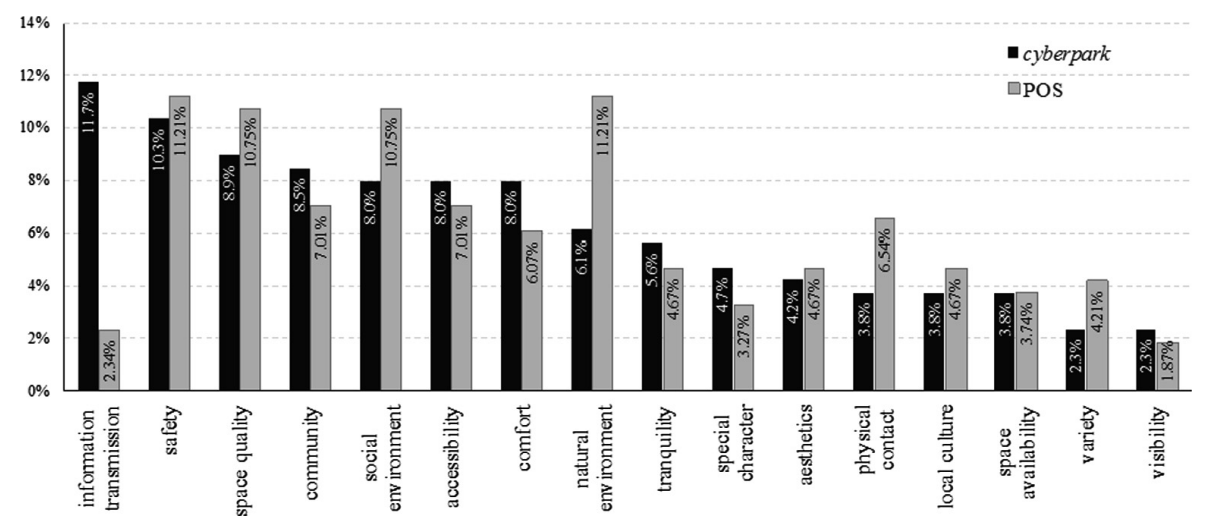

Fig. 5. Qualities of spaces

From a list of facilities that public spaces could offer, experts were asked to choose those that are most necessary to cyberpark and to POS. As Fig. 6 illustrates, internet facilities, interactive information and places to work are essential for cyberpark, and of rather low importance to POS. In turn, places to sit and facilities for visitors in general, including facilities for specific groups (such as disadvantaged citizens or children), are regarded as most important for POS. 


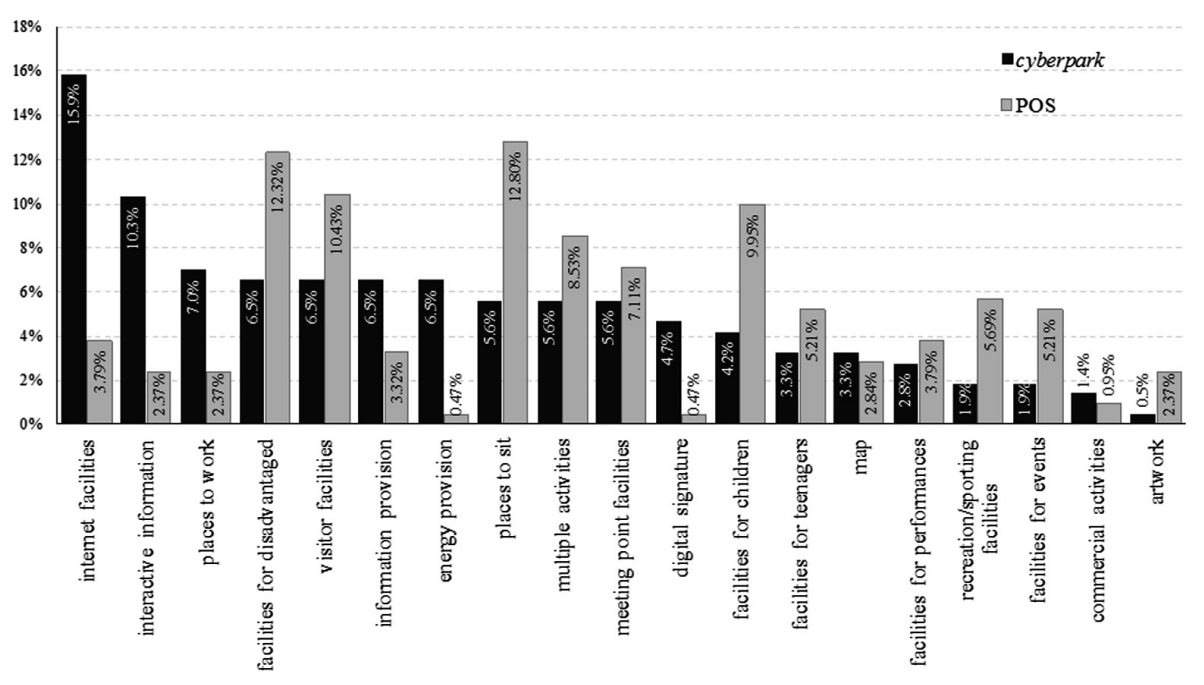

Fig. 6. Facilities provided

Turning to the activities that the public spaces under examination should facilitate, experts indicated that relaxing, socializing and communicating with people constitute the most important functions that a cyberpark should offer (Fig. 7). The first two are also the two main activities that POS also facilitate, along with strolling and leisure. Interestingly, educational, work and communicate facilities are ranked quite high in cyberpark (fourth, fifth and third, respectively), but very low in POS, which are mainly appreciated for their recreational and leisure role.

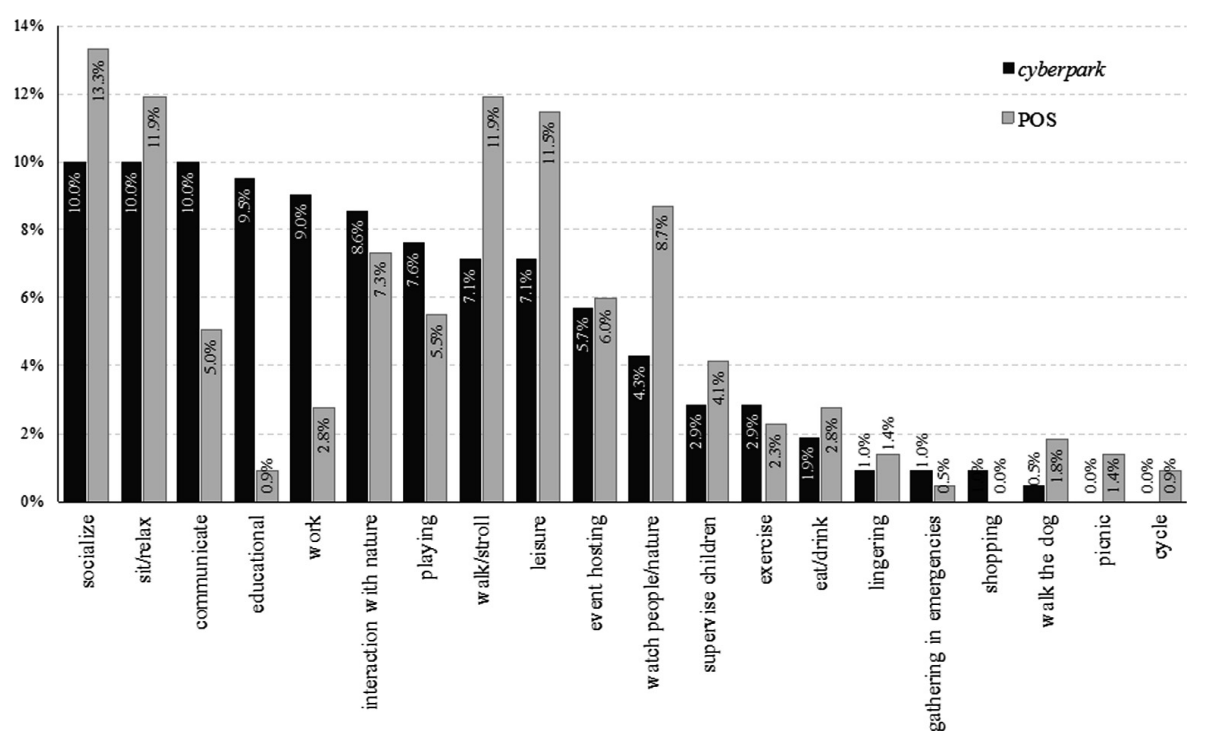

Fig. 7. Facilitated activities 
The next question asked respondents to assess what types of places are best for each of the spaces examined. Figure 8 presents the findings. We see that mainly parks, followed by squares, historic sites and river banks and canals, are the most suitable places for deploying both cyberpark and POS, whereas cemeteries, parking-spaces and sport-fields are the least appropriate for both. Spaces that are regarded as suitable to host a cyberpark, but are not that appropriate for POS, are leftover or vacant spaces (ranked fifth), as well as bus and railway stops and stations (ranked eight). In turn, experts believe that places which could better accommodate a cyberpark, rather than conventional POS, are institutional public spaces (like, university campuses, libraries, church and school yards), internalized 'public' spaces (such as, interior of shopping/leisure centres and retail space), and any other space with public access. We note with interest that CyberParks experts do not confine cyberpark to outdoor locations.

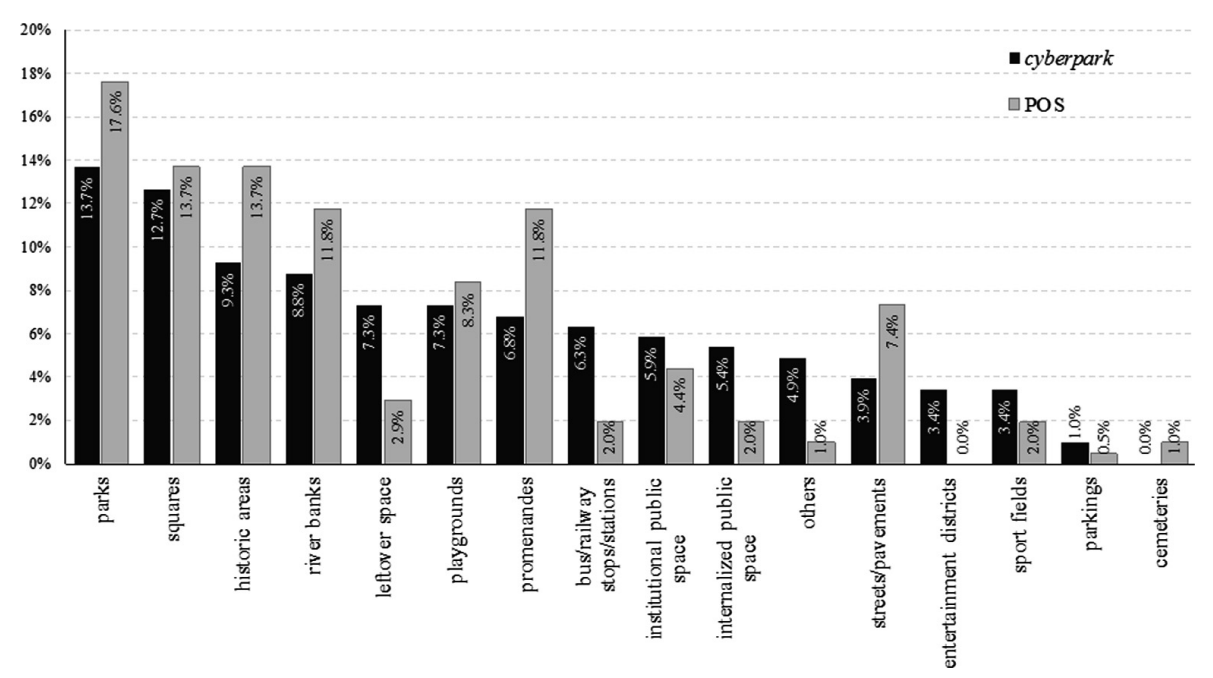

Fig. 8. Suitable spaces

Next, we asked the specialists to specify, which locations are best for a cyberpark vis-à-vis POS. As Fig. 9 illustrates, city centre and downtown are most appropriate places for cyberpark. City centre is also a good location for POS in general; however, downtown locations are not regarded as suitable. Third in line comes sites within neighbourhoods, which is seen as a quite good location for the development of all POS as well. 


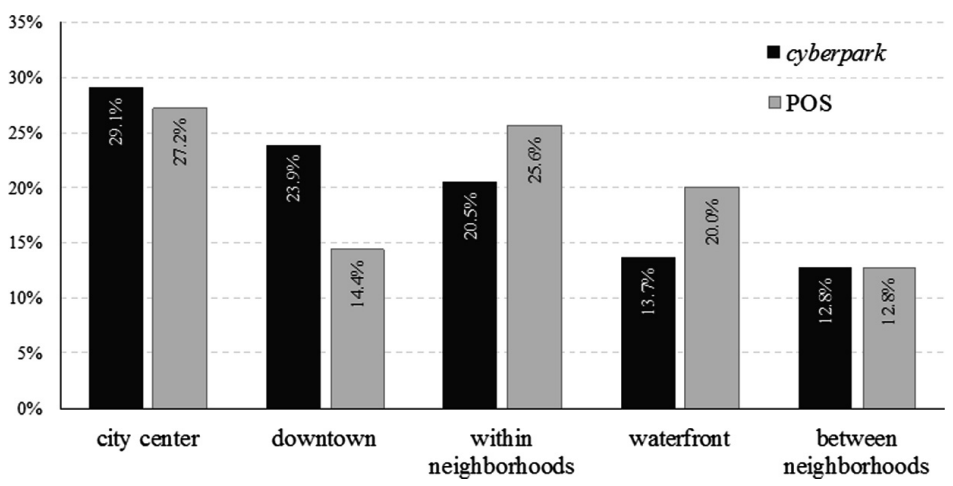

Fig. 9. Suitable locations

The appropriate size of both cyberpark and conventional POS was another issue explored with the experts. As Fig. 10 makes evident, medium and small size spaces are most appropriate for a cyberpark, whereas for POS medium to large size should be sought. Of course, what constitutes small or large size of space is a relative and rather subjective measure (which might differ between different disciplines or exerts), however the answers we received provide an indication that size could be a characteristic that distinguishes cyberpark from conventional POS.

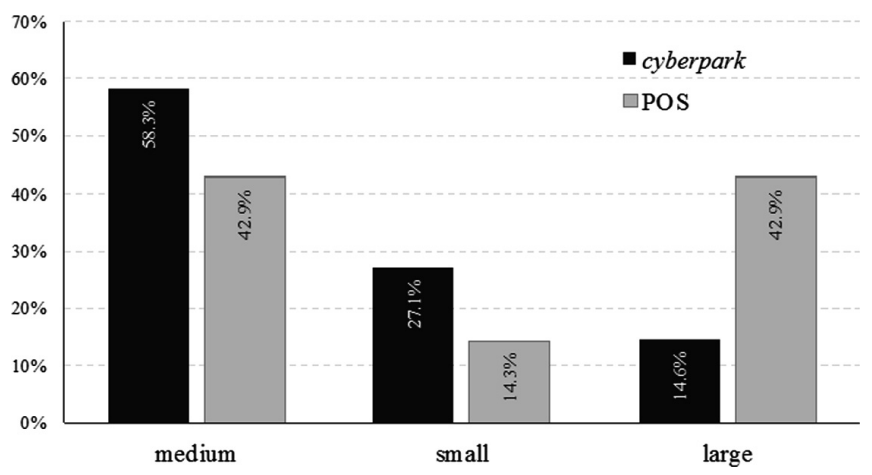

Fig. 10. Suitable sizes

Figure 11 presents the results concerning the target population of both cyberpark and POS. We see that city dwellers are the main target group of both spaces. Moreover, a cyberpark should focus on servicing tourists and visitors (ranked second), and the youth (ranked third), whereas POS should primarily target local residents living nearby. Intriguingly, we also see that elderly people is a legitimate target group for POS, but are not regarded that can take advantage of the special facilities that a cyberpark can offer. 


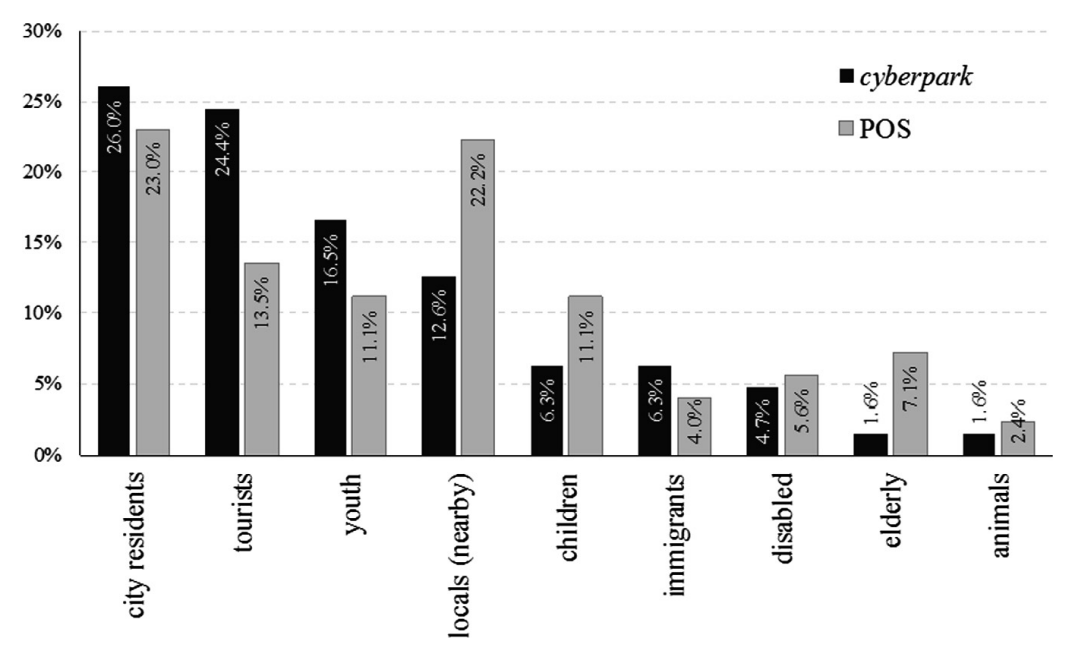

Fig. 11. Target population

The development and maintenance of public space constitutes a major issue, especially during the times of economic crisis, where local authorities are facing increased financial problems and difficulties. The question that follows examines who the experts reckon should bear the costs of providing and maintaining the spaces under investigation. Four options were given: the users of the spaces, all citizens, the local authorities with the municipality, and the private sector. As Fig. 12 depicts, the vast majority of experts agree that local authorities should undertake such costs, for the development and maintenance of both cyberpark and POS. However, in contrast to POS, experts seem to be open to other sources of finance when the question turns to cyberpark. Interestingly the $13.6 \%$ of the respondents regard that the private sector could contribute to the provision and maintenance of cyberpark and $9.1 \%$ is willing the users themselves to bear part of these costs.

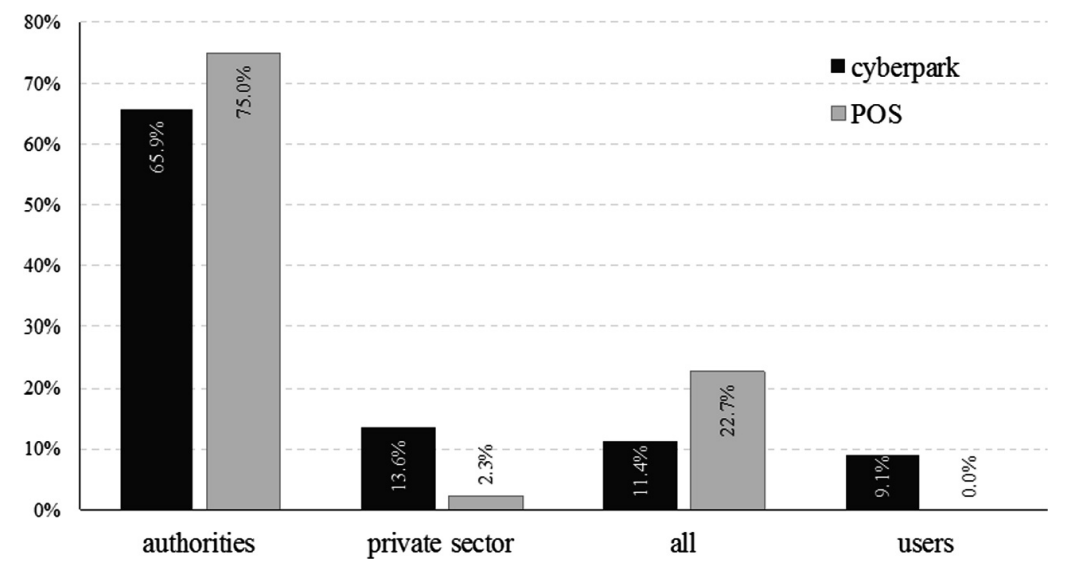

Fig. 12. Provision and maintenance 
The final set of questions explored the views of experts on various characteristics of cyberpark. In particular, the participants were asked to evaluate a number of statements concerning the value and specific aspects of cyberpark development, using an elevenpoint Likert scale, ranging of 0 (strongly disagree) to 10 (strongly agree). The statements were the following: (1) cyberparks are essential to a city, (2) they are costly to create, (3) they are costly to maintain, (4) they increase the welfare of the citizens, (5) controlled access should be applied, (6) security cameras should be used, (7) entrance fees should be applied, and (8) the users should contribute financially to their creation and maintenance. Table 2 presents the results. We see that the majority of experts believe that cyberpark places are quite important to the city (mean: 6.7, with most of respondents favouring the highest values); although they are costly in their development and maintenance (means respectively 6.3 and 6.0) they increase substantially the citizens welfare (mean: 7.3). When the question turns to issues of security, experts seems to be somewhat divided; although many are sceptical on applying security cameras and access control (most respondents are favouring the lowest values), there are a few who feel that such an approach would overall benefit cyberpark spaces (as the high standard deviation reveals). As regards the application of entrance fees and financial contribution by the users, respondents are rather reserved to both (means 1.5 and 2.1, respectively), corroborating the previous finding that cyberpark is essentially a public good and the local authorities should be those to bear its costs.

Table 2. Views on aspects of cyberpark provision

\begin{tabular}{|c|c|c|c|c|c|c|c|c|c|c|c|c|c|c|}
\hline & $\begin{array}{l}0 \\
(\%)\end{array}$ & $\begin{array}{l}1 \\
(\%)\end{array}$ & $\begin{array}{l}2 \\
(\%)\end{array}$ & $\begin{array}{l}3 \\
(\%)\end{array}$ & $\begin{array}{l}4 \\
(\%)\end{array}$ & \begin{tabular}{|l|}
5 \\
$(\%)$
\end{tabular} & \begin{tabular}{|l|}
6 \\
$(\%)$
\end{tabular} & \begin{tabular}{|l|}
7 \\
$(\%)$
\end{tabular} & $\begin{array}{l}8 \\
(\%)\end{array}$ & $\begin{array}{l}9 \\
(\%)\end{array}$ & $\begin{array}{l}10 \\
(\%)\end{array}$ & $\mathrm{N}$ & Mean & $\begin{array}{l}\text { Stand. } \\
\text { dev. }\end{array}$ \\
\hline $\begin{array}{l}\text { Essential to a } \\
\text { city }\end{array}$ & 0 & 0 & 2.4 & 2.4 & 7.1 & 28.6 & 7.1 & 7.1 & 28.6 & 2.4 & 14.3 & 42 & 6.7 & 2.1 \\
\hline Costly to create & 0 & 2.4 & 4.9 & 9.8 & 7.3 & 22.0 & 7.3 & 7.3 & 9.8 & 17.1 & 12.2 & 41 & 6.3 & 2.6 \\
\hline $\begin{array}{l}\text { Costly to } \\
\text { maintain }\end{array}$ & 0 & 0 & 11.9 & 4.8 & 7.1 & 19.0 & 11.9 & 16.7 & 16.7 & 0 & 11.9 & 42 & 6.0 & 2.4 \\
\hline Increase welfare & 0 & 0 & 0 & 2.4 & 2.4 & 14.6 & 17.1 & 14.6 & 22.0 & 7.3 & 19.5 & 41 & 7.3 & 1.9 \\
\hline $\begin{array}{l}\text { Control access } \\
\text { should be } \\
\text { applied }\end{array}$ & 16.7 & 9.5 & 16.7 & 2.4 & 2.4 & 19.0 & 7.1 & 4.8 & 7.1 & 9.5 & 4.8 & 42 & 4.2 & 3.3 \\
\hline $\begin{array}{l}\text { Security cameras } \\
\text { should be } \\
\text { applied }\end{array}$ & 23.3 & 11.6 & 2.3 & 2.3 & 2.3 & 18.6 & 7.0 & 2.3 & 14.0 & 7.0 & 9.3 & 43 & 4.5 & 3.6 \\
\hline $\begin{array}{l}\text { Entrance fees } \\
\text { should be } \\
\text { applied }\end{array}$ & 54.8 & 19.0 & 0 & 2.4 & 7.1 & 11.9 & 0 & 2.4 & 0 & 2.4 & 0 & 42 & 1.5 & 2.3 \\
\hline $\begin{array}{l}\text { Users should } \\
\text { contribute } \\
\text { financially }\end{array}$ & 46.3 & 12.2 & 7.3 & 7.3 & 7.3 & 2.4 & 12.2 & 0 & 2.4 & 2.4 & 0 & 41 & 2.1 & 2.6 \\
\hline
\end{tabular}




\section{On Cyberpark and Public Spaces}

As mentioned above, the CyberParks Action defines cyberpark as “... a new type of urban landscape where nature and cyber technologies blend together to generate hybrid experiences and enhance quality of life. ... ICT can be used in this context to give or gather information, to aid co-creation of space, to allow crowd sourcing of information and opinions, and to allow effective sharing or self-monitoring of activities" (Agora 2017). This conception underlines the unique character of a cyberpark, functioning as a distinct entity and offering more than the mere sum of its major components, i.e. 'cyber' and 'parks'. Having this in mind, it is worth probing into the views of CyberParks participants ${ }^{2}$ as concerns the main characteristics, conditions and functions of this new hybrid space. Such an exercise is quite important for the formation of a theoretical notion that aspires to adequately persuade policy makers, local authorities, and the public, in order to be effectively implemented and well accepted by stakeholders.

Bearing the above in mind, it is useful to distinguish the "points of agreement", or "strong points" of experts concerning the notion of cyberpark, and compare it with equivalent points concerning POS in general. As "points of agreement" or "strong points" we refer to the responses of various questions, which scored $20.0 \%$ or more in frequency, compared to the rest of the responses to the specific question. For cyberpark, these were:

- Importance of constituent elements: people (59.1\%)

- Cyberpark keywords: Technology/ICT (43.4\%), Space/POS (26.4\%)

- Suitable locations: city centre $(29.1 \%)$

- Suitable sizes: medium $(58.3 \%)$

- Target Population: city residents (26.0\%), tourists $(24.9 \%)$

- Provision and maintenance: authorities (65.9\%).

In the categories of Qualities of space, Facilities provided, Facilitated activities, and Suitable spaces there were no responses which clearly dominated the opinions of experts.

At the same time, looking at the equivalent responses for the POS, the same categories of questions gather high percentages of most preferred responses, and furthermore, the first preferences of these categories were the same as in cyberpark. More specifically:

- Importance of constituent elements: people (68.2\%)

- Suitable locations: city centre (27.2\%)

- Suitable sizes: medium $(42.9 \%)$

${ }^{2}$ All CyberParks participants are considered, by definition, to be experts on the issues examined here (i.e. the relationship between POS and ICTs), since this is a prerequisite form their participation in the COST Action. However, as discussed, CyberParks participants might understand and approach cyberpark slightly different, due to their different scientific discipline, theoretical background, occupation, nationality, etc. 
- Target Population: city residents (23\%), locals (22.2\%), which is almost double than tourists $(13.5 \%)$

- Provision and maintenance: authorities (65.9\%).

In Qualities of space, Facilities provided, Facilitated activities, and Suitable spaces there were no responses which clearly dominated the opinions of experts (as was the case in cyberpark).

The category of Cyberpark keywords had no equivalent in POS. One could note, though, a sign of contradiction in experts' responses in this category, compared to the ones in Importance of constituent elements. So, although people are recognized as the most important element for cyberpark, in comparison to space and technology, the most "favourite" keywords belong to the categories of technology (ICT) and space $(P O S)$. At the same time, keywords of the category of technology gathered 61 preferences, the space category 54, and the category of people 45 preferences.

In the categories of no responses of high occurrence (Qualities of space, Facilities provided, Facilitated activities, and Suitable spaces), coincidence of the same answers in the first places of preferences in cyberpark and POS was recorded in Facilitated activities [socialize with $10 \%$ of opinions in cyberpark (sharing the first place with relax and communicate) and $13.3 \%$ in POS], and in Suitable spaces (Parks with $13.7 \%$ of opinions in cyberpark and $17.6 \%$ in POS), while in Qualities of space, safety was a close second (10.3\%) in cyberpark [first being information transmission (11.75\%)], and first (11.21\%) in POS (with the same percentage of opinions with natural environment).

Conclusions drawn from the above are that for experts, a cyberpark retains the "strong" characteristics of POS. It is worth noticing that, besides these common "strong" characteristics there were no other "strong" ones, either solely for a cyberpark or for POS. So, for experts, a cyberpark has to share significant common ground with POS, and ideally, both should have people as the most important constituent element, they should be in the city centre, being of a medium size, having city residents as their target group, and having authorities (municipality, etc.) responsible for their provision and maintenance. Experts also tend to think, in lower percentages, that both cyberparks and POS should be safe, with socializing as the most important facilitated activity, and preferably in parks.

Looking for elements distinguishing cyberpark space from conventional POS, there were no coincidences in the first three categories of facilities of high occurrence in responses between cyberpark and POS in the category of Facilities provided, and expectedly so - internet was the prime preference for the former with $15.9 \%$ of responses, with places to sit being the prime equivalent for the latter with $12.5 \%$ of responses. The responses of high occurrence in this category (internet, interacting information, and places to work for cyberpark, and places to sit, facilities for disadvantaged, and facilities for visitors for POS) show a differentiation of the character of cyberpark from this of POS, the former being more "informatic" with the latter being closer to social values and aspects. This is perhaps the reason why experts see suitable places for cyberparks to be leftover spaces, bus/railway stations and stops and 
internalised public spaces of rather medium to small sizes. Moving to the Accommodated activities, cyberpark's role is perceived in facilitating communication, education and work, whereas POS is appreciated mainly for its recreation and leisure aspects. As such, it is not surprising that in the categories of Target population, elderly people are almost ignored in cyberpark (presumably because this group is expected to use ICT at a lesser degree than young people ${ }^{3}$ ) whereas in Provision and maintenance, the private sector is seen as a possible provider of cyberpark in a much higher percentage that the equivalent in POS. This is also verified in the category of Cyberpark keywords where ICT, POS, interactive, and enhanced were considered as better characterizing a cyberpark. As discussed above, all these belong to the categories of technology, and space, while the category of people gathered keywords with lower preferences of the former.

\section{Conclusions}

This chapter comes to provide, in a more systematic and organized way, a contextualization of the various dimensions of the cyberpark concept, which emerge due to different perspectives, disciplines, backgrounds, etc. of the 84 experts involved in the CyberParks COST Action. We believe that such analysis would enrich the ongoing dialogue within the respective scientific community, and facilitate interactions between relevant, yet fragmented, research in various scientific areas and countries. From the analysis we conducted, a number of points drawn should be highlighted.

First, cyberpark is perceived as a specific type of POS, characterised by enhanced provision of information technology that advance modern life. However, as any type of POS, cyberpark is a space aiming to serve the needs of real people, by combining recreation and leisure along with connectivity, interaction and community development. Second, although most POS areas are suitable for the deployment of a cyberpark, such spaces might be more relevant in specific sites and locations that best serve its purpose. Leftover spaces, bus and railway stops and stations, institutional public spaces and internalized 'public' spaces in downtown and city centres seem to be appropriate such locations. Third, cyberpark, though a bit costly in development and maintenance (due to the ICT element that it adds to the conventional POS), are appreciated for adding value to modern cities. On these grounds, local authorities should opt for their provision and should bear these costs as it is argued that these cyberpark spaces would increase citizens' welfare and the quality of urban life overall.

\footnotetext{
${ }^{3}$ This might be due to lack of dexterity or need on the part of the elderly to use ICTs, at least as compared to the younger generations. Surely, it seems that technology, from computing to cell phones, is not designed having the elderly in mind.
} 


\section{References}

Agora Cyberparks (2017). http://cyberparks-project.eu/agora/forums/topic/extended-definitionbased-on-discussion/. Accessed June 2017

Smaniotto Costa, C., Martínez, A.B., Álvarez, F.J., Šuklje-Erjavec, I., Menezes, M., PallaresBarbera, M.: Digital tools for capturing user's needs on urban open spaces: drawing lessons from cyberparks project. In: Certomà, C., Dyer, M., Pocatilu, L., Rizzi, F. (eds.) Citizen Empowerment and Innovation in the Data-Rich City, pp. 177-194. Springer, Cham (2017). https://doi.org/10.1007/978-3-319-47904-0_11

Open Access This chapter is licensed under the terms of the Creative Commons Attribution 4.0 International License (http://creativecommons.org/licenses/by/4.0/), which permits use, sharing, adaptation, distribution and reproduction in any medium or format, as long as you give appropriate credit to the original author(s) and the source, provide a link to the Creative Commons license and indicate if changes were made.

The images or other third party material in this chapter are included in the chapter's Creative Commons license, unless indicated otherwise in a credit line to the material. If material is not included in the chapter's Creative Commons license and your intended use is not permitted by statutory regulation or exceeds the permitted use, you will need to obtain permission directly from the copyright holder. 